\title{
Mudanças nos compostos bioativos e atividade antioxidante de pimentas da região amazônica ${ }^{1}$
}

\author{
Ana Vânia Carvalho², Rafaella de Andrade Mattietto²,
} Alessandro de Oliveira Rios ${ }^{3}$, Karla Suzana Moresco ${ }^{3}$

\begin{abstract}
Changes in bioactive compounds and antioxidant activity of peppers from the Amazon region

Embrapa Amazônia Oriental owns and active bank of peppers with different genotypes of the genus Capsicum, which have not been analyzed for their functional characteristics and antioxidant capacity yet. This study aimed to determine the contents of ascorbic acid, phenolic compounds, total carotenoids and total antioxidant activity in immature and mature fruits of Capsicum peppers genotypes. The levels of vitamin C (100.76-

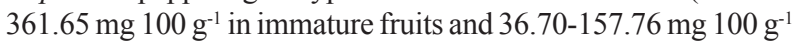
in mature fruits) decreased with fruit maturity. Total carotenoids were not detected in immature fruits, however, values of 73.80$1349.97 \mu \mathrm{g} \mathrm{g}^{-1}$ were observed for mature fruits, according to the genotype. The contents of phenolic compounds increased in mature fruits (147.40-718.64 mg GAE $100 \mathrm{~g}^{-1}$ ) for eight out of nine genotypes evaluated. The pepper fruits showed significant antioxidant activity $\left(55.02-92.03 \mu \mathrm{M}\right.$ trolox $\mathrm{g}^{-1}$ in immature fruits and $39.60-113.08 \mu \mathrm{M}$ trolox $\mathrm{g}^{-1}$ in mature fruits). It was concluded that the degree of fruit maturation affected the contents of bioactive compounds of the genotypes evaluated. IAN-186301 and IAN-186324, for total carotenoids; IAN-186301, IAN-186311, IAN-186312 and IAN-186313, for ascorbic acid; IAN-186304 and IAN-186311, for phenolic compounds; and IAN-186311, for antioxidant activity, were considered the most promising genotypes, with potential to be used in breeding programs.
\end{abstract}

KEY-WORDS: Capsicum L.; ascorbic acid; carotenoids; phenols.

\section{INTRODUÇÃO}

As espécies do gênero Capsicum L. são popularmente conhecidas como pimentas e pimentões. Esse gênero, originário do continente americano, pertence à família Solanaceae e apresenta ampla diversidade de espécies, incluindo quatro espécies domesticadas: C. annuит var. anпuит, C. baccatum var. pendulum,

\section{RESUMO}

A Embrapa Amazônia Oriental possui um Banco Ativo de Pimenteira com diferentes genótipos do gênero Capsicum, os quais ainda não foram analisados, quanto às suas características funcionais e capacidade antioxidante. Este estudo objetivou determinar os teores de ácido ascórbico, compostos fenólicos, carotenoides totais e a atividade antioxidante total, em frutos imaturos e maduros de genótipos de pimentas Capsicum spp. As concentrações de vitamina C (100,76-361,65 mg $100 \mathrm{~g}^{-1}$ nos frutos imaturos e $36,70-157,76 \mathrm{mg} 100 \mathrm{~g}^{-1}$ nos maduros) decresceram com a maturação dos frutos. Carotenoides totais não foram detectados nos frutos imaturos, porém, nos frutos maduros, observaram-se valores de 73,80-1349,97 $\mu \mathrm{g} \mathrm{g}^{-1}$, em função do genótipo. Os teores de compostos fenólicos aumentaram nos frutos maduros $\left(147,40-718,64 \mathrm{mg}\right.$ GAE $\left.100 \mathrm{~g}^{-1}\right)$, para oito dos nove genótipos avaliados. Os frutos de pimenteira apresentaram significativa atividade antioxidante $\left(55,02-92,03 \mu \mathrm{M}\right.$ trolox $\mathrm{g}^{-1}$ nos frutos imaturos e 39,60-113,08 $\mu \mathrm{M}$ trolox $\mathrm{g}^{-1}$ nos maduros). Concluiu-se que o grau de maturação dos frutos influenciou nos teores de compostos bioativos dos genótipos estudados. Destacaram-se, como genótipos promissores com potencial para serem utilizados em programas de melhoramento genético, IAN186301 e IAN-186324, pelos altos teores de carotenoides totais; IAN-186301, IAN-186311, IAN-186312 e IAN-186313, com relação às altas concentrações de ácido ascórbico; IAN-186304 e IAN-186311, pelos altos teores de compostos fenólicos; e IAN-186311, para atividade antioxidante.

PALAVRAS-CHAVE: Capsicum L.; ácido ascórbico; carotenoides; fenóis.

C. chinense e C. frutescens (Reifschneider 2000, Carvalho \& Bianchetti 2004).

As pimentas, além de micro e macronutrientes, possuem uma série de substâncias com propriedades antioxidantes, que podem ter impacto significativo na prevenção de doenças degenerativas, incluindo câncer, doenças cardiovasculares, cataratas e o funcionamento do sistema imune. Dentre tais substâncias,

1. Trabalho recebido em jan./2014 e aceito para publicação em nov./2014 ( $\mathrm{n}^{\circ}$ registro: PAT 28056).

2. Empresa Brasileira de Pesquisa Agropecuária (Embrapa Amazônia Oriental), Belém, PA, Brasil.

E-mails: ana-vania.carvalho@embrapa.br, rafaella.mattietto@embrapa.br.

3. Universidade Federal do Rio Grande do Sul (UFRGS), Instituto de Ciência e Tecnologia de Alimentos, Porto Alegre, RS, Brasil.E-mails: alessandro.rios@ufrgs.br, karlamoresco@gmail.com. 
destacam-se os compostos fenólicos, ácido ascórbico e carotenoides, constituintes cujos níveis podem variar de acordo com a espécie, genótipo e grau de maturação das pimentas (Howard et al. 2000, Marín et al. 2004, Davis et al. 2007, Ogiso et al. 2008).

Nos últimos anos, os compostos fenólicos têm atraído o interesse de pesquisadores, devido ao fato de mostrarem resultados promissores como poderosos antioxidantes, os quais podem proteger o corpo humano de radicais livres, cuja formação é associada ao metabolismo normal das células aeróbicas. A atividade antirradical livre dos compostos fenólicos é principalmente baseada nas propriedades redox de seus grupos hidroxilas e no relacionamento estrutural entre as diferentes partes de sua estrutura química (Burda \& Oleszek 2001, Materska \& Perucka 2005). Além disso, agem fortalecendo o sistema imunológico, regulando a expressão do gene, sinalização celular e metabolismo dos hormônios, influenciando na proliferação celular e apoptose (Finco et al. 2012).

O ácido ascórbico é uma vitamina hidrossolúvel e um importante antioxidante, que reage diretamente com o oxigênio simples, radical hidroxila e radical superóxido, além de regenerar a vitamina E (Carr \& Frei 1999). Segundo Reifschneider (2000), o gênero Capsicum é considerado boa fonte de ácido ascórbico. O conteúdo de ácido ascórbico encontrado nas pimentas brasileiras é de 52-134 mg $100 \mathrm{~g}^{-1}$, em frutos frescos. A pimenta Capsicum chinense (biquinho) contém $99 \mathrm{mg}$ de ácido ascórbico $100 \mathrm{~g}^{-1}$ de produto fresco, quantidade superior à necessidade diária de um indivíduo (60 $\left.\mathrm{mg} \mathrm{dia}^{-1}\right)$ (Lutz \& Freitas 2008).

Os carotenoides constituem uma classe de pigmentos naturais responsáveis pela diversidade de cor em frutas e hortaliças e podem ser encontrados em abundância nas pimentas. Tais compostos são antioxidantes (violaxantina, neoxantina e luteína), sendo que alguns também são pró-vitamínicos $\mathrm{A}$ $(\alpha-, \beta$ - e $\gamma$-caroteno, $\beta$-criptoxantina). Seus níveis podem variar entre genótipos e grau de maturidade e são influenciados pelas condições de cultivo e pelo processamento (Chuah et al. 2008, Menichini et al. 2009).

Nesse contexto, é importante conhecer como a maturação afeta a composição nutritiva e funcional dos frutos, pois sabe-se que há profunda mudança durante essa fase, ocorrendo a conversão de diversos pigmentos e constituintes existentes. Howard et al. (2000) estudaram os efeitos da maturação no teor de antioxidantes, em diferentes tipos de pimentas (C. annuum, $C$. frutescens e $C$. chinense), e constataram que a concentração de antioxidantes tende a aumentar, quando as pimentas alcançam a maturidade.

Embora alguns estudos tenham demonstrado que as pimentas possuem uma variedade de compostos bioativos (Howard et al. 2000, Deepa et al. 2007, Chuah et al. 2008, Alvarez-Parrilla et al. 2011, Zhuang et al. 2012), poucas espécies têm sido analisadas, com relação a esses importantes constituintes. Para a $C$. chinense, cujo centro de diversidade é a região amazônica, os estudos para identificação e quantificação de substâncias bioativas são ainda muito incipientes e praticamente inexistentes. Por muitos anos, as espécies amazônicas foram negligenciadas e, por isso, o seu conhecimento ainda é rudimentar. Além disso, as mudanças que ocorrem nos compostos bioativos durante a maturação e o efeito resultante na atividade antioxidante são informações que podem contribuir para uma melhor utilização e aproveitamento de diferentes tipos de pimenta.

A Embrapa Amazônia Oriental possui um Banco Ativo de Pimenteira com diferentes genótipos do gênero Capsicum, os quais ainda não foram analisados quanto às suas características funcionais $\mathrm{e}$ capacidade antioxidante. Assim, este estudo objetivou determinar os teores de ácido ascórbico, compostos fenólicos e carotenoides totais e a atividade antioxidante, em frutos imaturos e maduros de diferentes genótipos de pimentas Capsicum spp., bem como avaliar como as mudanças desses constituintes químicos influenciam na atividade antioxidante dos frutos.

\section{MATERIAL E MÉTODOS}

Pimentas de nove genótipos de Capsicum, provenientes do Banco Ativo de Germoplasma (BAG) da Embrapa Amazônia Oriental, em Belém (PA), foram colhidas no estádio imaturo (frutos fisiologicamente desenvolvidos, porém, sem mudança na cor da casca) e quando completamente maduras (frutos completamente desenvolvidos, com coloração da casca sem pontos verdes), de janeiro a dezembro de 2011.

O plantio dos genótipos estudados foi realizado mediante a semeadura em vasos de $20 \mathrm{~L}$, em cultivo protegido. Os experimentos foram instalados em julho de 2010 e a colheita dos frutos teve início após 6 meses do plantio. O clima do local é do tipo equatorial, com classificação climática Af, segundo Köppen-Geiger. O solo utilizado para enchimento dos 
vasos foi do tipo Latossolo Franco-Arenoso distrófico, adicionando-se cerca de $10 \%$ do total do vaso com esterco bovino. Foi realizada adubação, por ocasião do plantio e a cada 60 dias, com ureia, superfosfato triplo e cloreto de potássio ( $12,3 \mathrm{~g}, 4,8 \mathrm{~g}$ e $7,7 \mathrm{~g}$ por planta, respectivamente). A cada 30 dias, realizou-se adubação foliar à base de cálcio e boro (produto CalBor da Sanfol - Fortiplant; 2,5 $\mathrm{mL} \mathrm{L}^{-1}$ de água).

O plantio foi feito em delineamento em blocos casualizados, com três repetições, sendo cada bloco (repetição) composto por quatro plantas de cada genótipo. A identificação dos genótipos, bem como a coloração dos frutos quando imaturos e maduros, são apresentadas na Tabela 1.

Os frutos colhidos foram embalados em sacos de polietileno e armazenados em freezer $\left(-18^{\circ} \mathrm{C}\right)$. Para a realização das análises químicas dos frutos inteiros (casca, polpa e sementes), foi utilizado cerca de $1 \mathrm{~kg}$ de material. As amostras foram desintegradas em triturador de tecidos Turratec (TE-102, Tecnal, Piracicaba, Brasil), sendo todos os ensaios realizados em triplicata. A determinação da umidade foi realizada pela secagem do material em estufa, a $105^{\circ} \mathrm{C}$, até peso constante (AOAC 1997).

A extração e quantificação dos carotenoides totais foram realizadas segundo Godoy \& Rodriguez-Amaya (1994). As amostras foram misturadas com celite, com a finalidade de facilitar o rompimento das estruturas celulares e liberação dos carotenoides, e, em seguida, submetidas à extração com acetona P.A. $\mathrm{O}$ extrato foi transferido para um funil de separação contendo éter de petróleo (aproximadamente $50 \mathrm{ml}$ ) e a mistura lavada cinco vezes, com água destilada, para remoção da acetona. $\mathrm{O}$ extrato obtido foi filtrado em papel filtro contendo sulfato de sódio, para remoção da água, e, em seguida, transferido para um balão volu- métrico (100 ml), cujo volume foi ajustado com éter de petróleo. A solução foi analisada em espectrofotômetro (Thermo Scientific, modelo Evolution 300, EUA) por varredura, entre $350-700 \mathrm{~nm}$, para cada amostra.

O cálculo do teor de carotenoides totais foi realizado utilizando-se o valor de leitura no comprimento de onda que apresentou o maior pico, ou seja, para as pimentas IAN-186313, IAN-186335 e IAN-186304, a leitura foi efetuada no comprimento de onda de $470 \mathrm{~nm}$, que corresponde ao licopeno. Já para as demais amostras, o maior pico foi observado no comprimento de onda de $444 \mathrm{~nm}$, que corresponde ao $\alpha$-caroteno.

Os coeficientes de absorção para o licopeno e $\alpha$-caroteno em éter de petróleo são 3420 e 2800, respectivamente (Godoy \& Rodriguez-Amaya 1994). A fórmula utilizada para o cálculo foi CT $\left(\mu \mathrm{g} \mathrm{g}^{-1}\right)=$ $10^{4}$.abs.vol $/{ }_{1 \%} \mathrm{E}^{1 \mathrm{~cm}} \cdot \mathrm{m}$, em que $\mathrm{CT}=$ carotenoides totais; Abs = absorbância no maior pico detectado; Vol = volume do balão utilizado na diluição $(\mathrm{ml})$; ${ }_{1 \%} \mathrm{E}^{\mathrm{lcm}}=$ valores tabelados (absortividade em função do carotenoide predominante na amostra); $\mathrm{m}=$ massa da $\operatorname{amostra}(\mathrm{g})$.

O teor de ácido ascórbico foi determinado mediante titulação com 2,6-dicloroindofenol (DFI) $(0,02 \%)$ (AOAC 1984), substituindo-se o solvente extrator ácido metafosfórico por ácido oxálico. Os resultados foram expressos como $\mathrm{mg}$ de ácido ascórbico por $100 \mathrm{~g}$ de amostra, de acordo com a seguinte fórmula:

ácido ascórbico $\left(\mathrm{mg} 100 \mathrm{~g}^{-1}\right)=\frac{T}{(P / 3) \times m} \times 100$

em que $\mathrm{T}=$ volume de DCFI gasto na titulação da amostra (ml); $\mathrm{P}=$ volume de DCFI gasto na padronização $(\mathrm{ml}) ; \mathrm{m}=$ quantidade de amostra $(\mathrm{g})$.

Tabela 1. Identificação de nove genótipos de Capsicum spp. provenientes do Banco Ativo de Germoplasma da Embrapa Amazônia Oriental (Belém, PA, 2012).

\begin{tabular}{|c|c|c|c|c|}
\hline \multirow{2}{*}{ Código } & \multirow{2}{*}{ Espécie } & \multirow{2}{*}{ Nome popular } & \multicolumn{2}{|c|}{ Coloração do fruto } \\
\hline & & & Imaturo & Maduro \\
\hline IAN-186301 & Capsicum annuum L. & Pimenta PMO & Verde & Vermelha \\
\hline IAN-186304 & C. annuum L. var. annuum & Pimenta Carajás Vermelha & Verde & Vermelha \\
\hline IAN-186309 & C. chinense Jacq. & Pimenta Curuçazinho & Verde & Amarela \\
\hline IAN-186310 & C. chinense Jacq. & Pimenta Cumari do Pará & Verde & Amarela \\
\hline IAN-186311 & C. chinense Jacq. & Pimenta Murupi & Verde & Amarela \\
\hline IAN-186312 & C. annuum $\mathrm{L}$. & Pimenta Amarela & Verde & Amarela \\
\hline IAN-186313 & C. chinense Jacq. & Pimenta Biquinho & Verde & Vermelho-alaranjada \\
\hline IAN-186324 & Capsicum sp. & Pimenta Olho-de-mutum & Roxa & Vermelha \\
\hline IAN-186335 & Capsicum sp. & Pimenta de Bico & Verde & Vermelho-alaranjada \\
\hline
\end{tabular}


A quantificação de compostos fenólicos foi realizada por meio do reagente Folin-Ciocalteau, segundo metodologia de Singleton \& Rossi (1965) modificada por Georgé et al. (2005), na qual acetona $70 \%$ foi utilizada como solvente extrator. Diluiu-se a amostra em $25 \mathrm{ml}$ da solução extratora, agitou-se por 30 minutos e filtrou-se, recolhendo-se o filtrado. Adicionou-se 2,5 ml de solução de Folin-Ciocalteau a $10 \%$, em $0,5 \mathrm{ml}$ do extrato filtrado, e, após 2 minutos, adicionou-se $2 \mathrm{ml}$ de solução de carbonato de sódio $7,5 \%$. Após agitação em vortex, levou-se ao banho-maria a $50^{\circ} \mathrm{C}$, por 15 minutos, e, a seguir, a mistura foi imersa em banho de gelo, por 30 segundos.

A absorbância foi determinada utilizando-se espectrofotômetro (Thermo Scientific, modelo Evolution 300, EUA) regulado a um comprimento de onda de $760 \mathrm{~nm}$. Em paralelo, uma curva padrão de ácido gálico (GAE) foi elaborada com as seguintes concentrações: $20 \mathrm{mg} \mathrm{L}^{-1}, 40 \mathrm{mg} \mathrm{L}^{-1}, 60 \mathrm{mg} \mathrm{L}^{-1}$, $80 \mathrm{mg} \mathrm{L}^{-1}$ e $100 \mathrm{mg} \mathrm{L}^{-1}$, sendo que todos os pontos da curva passaram pelas mesmas etapas descritas para os extratos. Os resultados foram expressos em equivalentes de ácido gálico (gallic acid equivalents - GAE) por $100 \mathrm{~g}$ de amostra, de acordo com a equação $\mathrm{FT}=\mathrm{C}_{\mathrm{EB}} \times\left(\mathrm{D}_{\mathrm{EB}} / \mathrm{m}\right) \times 100$, em que $C_{E B}$ é a concentração de ácido gálico na solução de amostra $\left(\mathrm{mg} \mathrm{L}^{-1}\right)$ referente ao Extrato Bruto (EB); $D_{E B}$ a diluição da amostra, em litros, referente ao Extrato Bruto (EB); e $m$ a massa da amostra utilizada na extração, expressa em gramas.

Para a determinação da atividade antioxidante total (AAT), inicialmente, procedeu-se à extração de antioxidantes (Larrauri et al. 1997, com algumas modificações): a amostra fresca foi pesada $(10 \mathrm{~g}) \mathrm{em}$ tubos de centrífuga e extraída, sequencialmente, com $40 \mathrm{ml}$ de metanol/água $\left(50: 50, \mathrm{v} \mathrm{v}^{-1}\right)$, à temperatura ambiente, por 1 hora. Os tubos foram centrifugados a $20.000 \mathrm{xg}$ por 20 minutos e o sobrenadante recolhido. Adicionou-se $40 \mathrm{ml}$ de acetona/água $\left(70: 30, \mathrm{v} \mathrm{v}^{-1}\right)$ ao resíduo, em temperatura ambiente, por 1 hora, e, a seguir, centrifugou-se a amostra nas mesmas condições citadas anteriormente. Os extratos de metanol e acetona foram combinados e ajustados para $100 \mathrm{ml}$, com água destilada, e usados para determinar a atividade antioxidante, a qual foi baseada no método desenvolvido por Re et al. (1999), com modificações de acordo com Rufino et al. (2007).

O radical $\mathrm{ABTS}^{+}$foi produzido pela reação, no escuro e em temperatura ambiente, de $7 \mathrm{mM}$ de solução estoque de $\mathrm{ABTS}^{+}$com $145 \mathrm{mM}$ de perssulfato de potássio, com a reação sendo realizada 12-16 horas antes do uso. A solução $\mathrm{ABTS}^{+}$foi diluída com etanol até obter-se absorbância de $0,70 \pm 0,02$, a $734 \mathrm{~nm}$. Após pipetar $30 \mu \mathrm{L}$ de amostra ou do padrão trolox, adicionou-se $3 \mathrm{ml}$ de solução diluída de $\mathrm{ABTS}^{+}$, e as absorbâncias foram lidas após 6 minutos da mistura da reação, a $734 \mathrm{~nm}$. Soluções etanólicas com concentrações conhecidas de trolox foram usadas para a calibração e os resultados foram expressos em $\mu \mathrm{M}$ trolox $\mathrm{g}^{-1}$ fruto.

Os resultados foram submetidos à análise de variância e a comparação das médias efetuada pelo teste de Scott-Knott, a 5\%, utilizando-se o programa GENES (Cruz 2006). As análises de correlação de Pearson foram realizadas por meio do programa SigmaPlot versão 11.0.

\section{RESULTADOS E DISCUSSÃO}

As características químicas dos frutos de pimenteira foram influenciadas pelas variações intrínsecas do material genético, bem como pelo grau de maturação dos frutos (Tabela 2). Para a umidade (Tabela 3), observaram-se valores variando de $81,46 \%$ a $91,42 \%$, para as pimentas imaturas, e de $78,19 \%$ a $89,39 \%$, para as maduras. Com a maturação dos frutos, os valores de umidade decresceram, verificando-se diferença significativa entre os dois estádios de maturação, ou seja, para um mesmo genótipo, os frutos perderam umidade com o amadurecimento, à exceção do genótipo IAN-186312, devido, provavelmente, à ocorrência de transpiração, processo fisiológico normal, em que os frutos perdem água na forma de vapor (Chitarra \& Chitarra 2005).

Menichini et al. (2009), em estudo sobre a influência do amadurecimento no conteúdo de carotenoides totais de frutos de Capsicum chinense Jacq. cv. Habanero, relataram teores de $62,7 \mathrm{mg} 100 \mathrm{~g}^{-1} \mathrm{e}$ $362 \mathrm{mg} 100 \mathrm{~g}^{-1}$ de material fresco, respectivamente para os frutos imaturos e maduros. Os autores destacaram que a coloração intensa e característica de frutos Capsicum ocorre devido ao conteúdo de carotenoides, e que os mesmos são sintetizados principalmente durante o amadurecimento dos frutos, o que pode justificar a não detecção desses compostos nos genótipos de pimenta imaturos deste trabalho. O mesmo foi relatado em outros estudos (Márkus et al. 1999, Howard et al. 2000, Deepa et al. 2007), nos quais os autores relacionaram o aumento no conteúdo de carotenoides e a mudança na cor dos frutos à 
Tabela 2. Comparação de médias entre nove genótipos e dois graus de maturação e desdobramentos da interação entre esses dois fatores nas variáveis umidade, carotenoides totais, ácido ascórbico, fenóis totais e atividade antioxidante total (AAT), em frutos de pimenteira (Capsicum spp.) (Belém, PA, 2012).

\begin{tabular}{|c|c|c|c|c|c|}
\hline Tratamento & $\begin{array}{l}\text { Umidade } \\
\left({\left.\mathrm{g} 100 \mathrm{~g}^{-1}\right)}\right.\end{array}$ & $\begin{array}{l}\text { Carotenoides totais } \\
\left(\mu \mathrm{g} \mathrm{g}^{-1}\right)\end{array}$ & $\begin{array}{c}\text { Ácido ascórbico } \\
\left(\mathrm{mg} 100 \mathrm{~g}^{-1}\right)\end{array}$ & 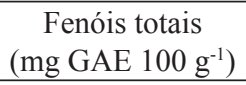 & $\begin{array}{c}\text { AAT } \\
\left(\mu \mathrm{M} \text { trolox } g^{-1}\right) \\
\end{array}$ \\
\hline \multicolumn{6}{|l|}{ Genótipo } \\
\hline IAN-186301 & $79,90 \mathrm{f}$ & $532,18 \mathrm{~b}$ & $168,52 \mathrm{~b}$ & $493,32 \mathrm{~b}$ & $80,28 \mathrm{~b}$ \\
\hline IAN-186304 & $83,06 \mathrm{e}$ & $248,52 \mathrm{c}$ & $119,46 \mathrm{c}$ & $463,16 \mathrm{c}$ & $74,73 \mathrm{c}$ \\
\hline IAN-186309 & $84,43 \mathrm{~d}$ & $36,90 \mathrm{f}$ & $94,41 \mathrm{~d}$ & $414,34 \mathrm{~d}$ & $56,69 \mathrm{~d}$ \\
\hline IAN-186310 & $85,03 \mathrm{~d}$ & $29,93 \mathrm{~g}$ & $100,47 \mathrm{~d}$ & $304,01 \mathrm{f}$ & $49,76 \mathrm{~d}$ \\
\hline IAN-186311 & $86,59 \mathrm{c}$ & $39,78 \mathrm{f}$ & $177,92 \mathrm{~b}$ & $819,79 \mathrm{a}$ & $99,63 \mathrm{a}$ \\
\hline IAN-186312 & $89,81 \mathrm{a}$ & $45,63 \mathrm{f}$ & $167,19 \mathrm{~b}$ & $382,09 \mathrm{e}$ & $59,29 \mathrm{~d}$ \\
\hline IAN-186313 & $87,81 \mathrm{~b}$ & $104,23 \mathrm{~d}$ & $231,52 \mathrm{a}$ & $202,21 \mathrm{~g}$ & $55,61 \mathrm{~d}$ \\
\hline IAN-186324 & $87,24 \mathrm{~b}$ & 674,99 a & $110,66 \mathrm{c}$ & $382,46 \mathrm{e}$ & $72,96 \mathrm{c}$ \\
\hline IAN-186335 & $85,98 \mathrm{c}$ & $90,96 \mathrm{e}$ & $101,82 \mathrm{~d}$ & $123,44 \mathrm{~h}$ & $54,45 \mathrm{~d}$ \\
\hline Teste F & $78,64 * *$ & $1.405,80 * *$ & $219,63 * *$ & $967,69 * *$ & $173,04 * *$ \\
\hline \multicolumn{6}{|c|}{ Grau de maturação } \\
\hline Imaturo & 87,60 & 0,00 & 181,02 & 312,48 & 68,85 \\
\hline Maduro & 83,47 & 400,69 & 101,64 & 484,14 & 65,23 \\
\hline Teste $\mathrm{F}$ & $360,60 * *$ & $27.780,61 * *$ & $1.404,64 * *$ & $1.631,65 * *$ & $17,02 *$ \\
\hline
\end{tabular}

Médias seguidas das mesmas letras, na coluna, constituem grupo estatisticamente homogêneo, a $5 \%$, pelo teste de Scott-Knott. * e **: significativo a $5 \%$ e a $1 \%$, respectivamente.

Tabela 3. Comparação entre médias nos desdobramentos de genótipos, dentro de graus de maturação e graus de maturação dentro de genótipos, nas variáveis umidade, atividade antioxidante total (AAT), ácido ascórbico, fenóis totais e carotenoides totais, em frutos de pimenteira (Capsicum spp.) (Belém, PA, 2012).

\begin{tabular}{|c|c|c|c|c|}
\hline \multirow{2}{*}{ Genótipo } & \multicolumn{2}{|c|}{ Umidade $\left(\mathrm{g} 100 \mathrm{~g}^{-1}\right)$} & \multicolumn{2}{|c|}{$\operatorname{AAT}\left(\mu \mathrm{M}\right.$ trolox $\left.\mathrm{g}^{-1}\right)$} \\
\hline & Imaturo & Maduro & Imaturo & Maduro \\
\hline IAN-186301 & $81,46 \pm 1,49$ e $\mathrm{A}$ & $78,19 \pm 1,55 \mathrm{~d} \mathrm{~B}$ & $76,97 \pm 1,34 \mathrm{c} \mathrm{B}$ & $83,59 \pm 0,73 \mathrm{~b} \mathrm{~A}$ \\
\hline IAN-186304 & $84,66 \pm 0,98 \mathrm{~d} A$ & $81,47 \pm 1,20$ c B & $92,03 \pm 1,11$ a A & $57,42 \pm 2,71 \mathrm{~d} B$ \\
\hline IAN-186309 & $87,09 \pm 0,08 \mathrm{~b} \mathrm{~A}$ & $81,77 \pm 0,14$ c B & $55,02 \pm 2,34$ e A & $58,36 \pm 2,85 \mathrm{dA}$ \\
\hline IAN-186310 & $86,37 \pm 0,67$ c $A$ & $83,70 \pm 0,76 \mathrm{~b} \mathrm{~B}$ & $55,22 \pm 1,65$ e $A$ & $44,29 \pm 2,76$ e $B$ \\
\hline IAN-186311 & $88,38 \pm 0,23 \mathrm{~b} \mathrm{~A}$ & $84,79 \pm 0,06$ b B & $86,17 \pm 1,99$ b B & $113,08 \pm 4,01$ a A \\
\hline IAN-186312 & $90,38 \pm 0,41$ a $\mathrm{A}$ & $89,39 \pm 0,54$ a A & $56,18 \pm 2,13$ e $\mathrm{B}$ & $62,39 \pm 1,41 \mathrm{~d} A$ \\
\hline IAN-186313 & $91,42 \pm 0,51$ a $A$ & $84,20 \pm 0,78 \mathrm{~b} \mathrm{~B}$ & $60,85 \pm 3,96$ e $A$ & $50,36 \pm 1,08$ e B \\
\hline IAN-186324 & $90,47 \pm 0,59$ a $\mathrm{A}$ & $84,01 \pm 0,34 \mathrm{~b} \mathrm{~B}$ & $67,93 \pm 4,08 \mathrm{~d} B$ & $77,99 \pm 1,00 \mathrm{c} \mathrm{A}$ \\
\hline IAN-186335 & $88,24 \pm 0,34 \mathrm{~b} \mathrm{~A}$ & $83,72 \pm 1,45$ b B & $69,30 \pm 1,39 \mathrm{dA}$ & $39,60 \pm 2,25 \mathrm{fB}$ \\
\hline \multirow{2}{*}{ Genótipo } & Ácido ascórbico & \multicolumn{2}{|c|}{ Fenóis totais (mg GAE $\left.100 \mathrm{~g}^{-1}\right)$} & Carotenoides totais $\left(\mu \mathrm{g} \mathrm{g}^{-1}\right)$ \\
\hline & Imaturo & Imatur & Maduro & Maduro \\
\hline
\end{tabular}

\begin{tabular}{llllll}
\hline IAN-186301 & $212,97 \pm 3,26$ b A & $124,08 \pm 0,55$ b B & $268,00 \pm 27,54$ d B & $718,64 \pm 36,36$ b A ND a B & $1.064,35 \pm 19,38$ b A
\end{tabular}

IAN-186304 202,21 $\pm 15,23$ b A $\quad 36,70 \pm 0,01$ e B $\quad 411,15 \pm 10,47$ c B $\quad 515,16 \pm 2,07$ d A ND a B $\quad 497,04 \pm 15,74$ c A

IAN-186309 $100,76 \pm 0,01 \mathrm{dA} \quad 88,05 \pm 0,03 \mathrm{~dB} \quad 570,70 \pm 21,99$ a A $\quad 257,98 \pm 0,97 \mathrm{~g}$ B $\quad$ ND a B $\quad 73,80 \pm 2,93 \mathrm{~g} \mathrm{~A}$

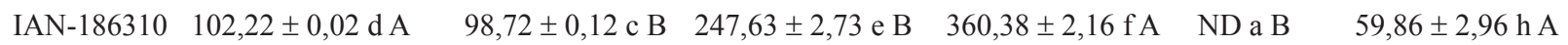

IAN-186311 $198,07 \pm 4,98$ b A $\quad 157,76 \pm 0,71$ a B $\quad 536,07 \pm 2,74$ b B $\quad 1103,5 \pm 3,13$ a A ND a B $\quad 79,56 \pm 7,12$ g A

IAN-186312 $207,82 \pm 0,08$ b A $\quad 126,56 \pm 0,01$ b B $\quad 287,63 \pm 1,18$ d B $\quad 476,54 \pm 19,93$ e A ND a B $\quad 91,26 \pm 8,59 \mathrm{fA}$

IAN-186313 $361,65 \pm 27,15$ a A $\quad 101,39 \pm 0,18$ c B $\quad 188,68 \pm 17,57 \mathrm{fB} \quad 215,73 \pm 0,01 \mathrm{~h} \mathrm{~A} \quad \mathrm{ND}$ a B $\quad 208,45 \pm 12,65 \mathrm{dA}$

IAN-186324 $138,77 \pm 0,09 \mathrm{c} \mathrm{A} \quad 82,55 \pm 1,82 \mathrm{~dB} \quad 203,02 \pm 38,51 \mathrm{fB} \quad 561,90 \pm 0,08 \mathrm{c} \mathrm{A} \quad$ ND a B $\quad 1.349,97 \pm 1,43$ a A

IAN-186335 $104,67 \pm 1,07 \mathrm{~d}$ A $\quad 98,97 \pm 0,21 \mathrm{c} \mathrm{B} \quad 99,48 \pm 1,91 \mathrm{~g} \mathrm{~B} \quad 147,40 \pm 0,91$ i A $\quad$ ND a B $\quad 181,92 \pm 12,95$ e A

Resultados apresentados em base seca (média \pm desvio-padrão). Médias seguidas de mesma letra minúscula (efeito dos genótipos), nas colunas, e letra maiúscula (efeito dos estádios de maturação), nas linhas, para cada característica avaliada, constituem grupo estatisticamente homogêneo, pelo Teste de Scott-Knott, a 5\%. AAT = atividade antioxidante total; $\mathrm{ND}=$ não detectado pela metodologia empregada. 
maturação dos mesmos, o que corrobora o observado no presente estudo.

Em relação aos carotenoides totais, para os genótipos de pimenta maduros avaliados, observou-se alta variabilidade, com valores de 59,86-1349,97 $\mu \mathrm{g} \mathrm{g}^{-1}$ (Tabela 3). As pimentas IAN$186324\left(1349,97 \mu \mathrm{g} \mathrm{g}^{-1}\right)$, IAN-186301 (1064,35 $\left.\mu \mathrm{g} \mathrm{g}^{-1}\right)$ e IAN-186304 (497,04 $\left.\mu \mathrm{g} \mathrm{g}^{-1}\right)$ se destacaram por apresentarem os maiores valores, o que indica tais genótipos como potenciais para serem selecionados em trabalhos de melhoramento genético, para obtenção de variedades com alto conteúdo de carotenoides totais. Já para a pimenta IAN-186310, observou-se o menor valor.

Topuz \& Ozdemir (2007), avaliando cinco cultivares de $C$. annuum L., determinaram carotenoides totais na faixa de $1440-2390 \mu \mathrm{g} \mathrm{g}^{-1}$, valores superiores aos verificados para as pimentas avaliadas neste trabalho. Collera-Zúñiga et al. (2005), em estudo comparativo da composição de carotenoides em três variedades de pimentas mexicanas $C$. annuиm L., verificaram, para carotenoides totais, valores de 67,6-75,2 $\mu \mathrm{g} \mathrm{g}^{-1}$. Em estudo sobre a caracterização e quantificação de constituintes antioxidantes de pimenta doce $(C$. annuum $\mathrm{L}$.), os autores verificaram $455,9 \mu \mathrm{g} \mathrm{g}^{-1}$ de carotenoides totais, para as pimentas maduras in natura (Marín et al. 2004), valor dentro da faixa observada neste estudo. Já em trabalho realizado por Zhuang et al. (2012), sobre as características bioativas e atividade antioxidante de nove pimentas, observou-se variação de carotenoides totais de $85,32-$ $1414,78 \mu \mathrm{g} \mathrm{g}^{-1}$ de peso fresco.

Em relação aos valores encontrados para ácido ascórbico, constatou-se variação entre os genótipos de pimenta imaturos de $100,76 \mathrm{mg}_{100 \mathrm{~g}^{-1} \mathrm{a}}$

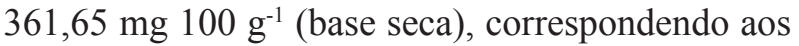
genótipos IAN-186309 e IAN-186313, respectivamente. Para os frutos maduros, essa faixa variou

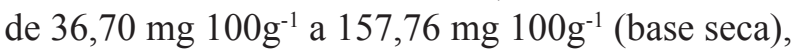
referentes aos genótipos IAN-186304 e IAN-186311, respectivamente.

Constatou-se que o processo de amadurecimento das pimentas contribuiu para o decréscimo nos níveis de ácido ascórbico, comportamento observado em todos os genótipos estudados, sendo as diferenças encontradas significativas (Tabelas 2 e 3 ). A mesma observação foi evidenciada por Deepa et al. (2007), estudando dez genótipos de Capsicum provenientes da Índia, que, igualmente, expressaram seus resultados em base seca, com valores para o estádio imaturo de 58,8-200 mg $100 \mathrm{~g}^{-1}$ e maduro de
64-220 mg $100 \mathrm{~g}^{-1}$. Entretanto, Howard et al. (2000) e Zhuang et al. (2012), estudando variedades de C. frutescens, $C$. annuum e $C$. chinense, concluíram que os teores de vitamina $\mathrm{C}$, em pimentas maduras, podem ser maiores ou permanecerem constantes, em relação às pimentas imaturas, fato inverso ao observado neste trabalho.

Durante o amadurecimento, o teor de ácido ascórbico aumenta nos estádios iniciais de desenvolvimento, até a maturação total, mas, quando excessivamente maduro, esse conteúdo diminui significativamente. Esse fato ocorre em função da desorganização da parede celular, levando à oxidação do ácido ascórbico, provavelmente devido à ação das enzimas polifenoloxidase e ácido ascórbico oxidase (Vazquez-Ochoa \& Colinas-Leon 1990).

Quanto aos compostos fenólicos, observou-se, para os genótipos de pimenta no estágio ima-

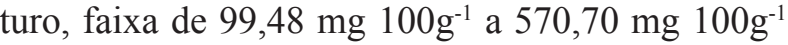
(base seca), referente aos genótipos IAN-186335 e IAN-186309, respectivamente. Houve diferença significativa $(p \leq 0,05)$ entre todos os genótipos de pimenta madura estudados, constatando-se valores de $147,40 \mathrm{mg} 100 \mathrm{~g}^{-1}$ a $1.103,50 \mathrm{mg} 100 \mathrm{~g}^{-1}$, obtidos para os genótipos IAN-186335 e IAN-186311, respectivamente.

Em relação ao comportamento frente ao amadurecimento, constatou-se que todos os genótipos apresentaram aumento significativo $(\mathrm{p} \leq 0,05)$ de compostos fenólicos, quando maduros, exceto o genótipo IAN-186309, que apresentou decréscimo de $54,79 \%$ no teor, quando comparado ao estádio imaturo. Possivelmente, esse aumento nos teores de compostos fenólicos nos frutos maduros seja devido ao fato de que esses compostos são, em grande parte, responsáveis pela coloração e sabor da grande maioria dos frutos (Kays 1991). Assim, os fenóis e seus derivados são componentes significativos do gosto e odor, estando envolvidos, também, nas reações de escurecimento. Segundo Chitarra (1997), os compostos fenólicos são influenciados por fatores como variedade, maturação, nutrição mineral e condições edafoclimáticas, desempenhando importante papel na determinação do sabor dos frutos.

Howard et al. (2000), avaliando compostos fenólicos em pimentas $C$. annuиm, C. frutescens e C. chinense, por dois diferentes métodos de determinação (Folin-Ciocalteu e HPLC), observaram que, em geral, os teores aumentaram com a maturação, independentemente do método de determinação aplicado. 
Assim como neste trabalho, os autores concluíram que apenas para uma cultivar (C. annuum - Yellow Bell) o teor de compostos fenólicos decresceu com a maturação. Segundo os autores, o teor mais baixo de compostos fenólicos observado para a citada cultivar, com a maturação dos frutos, foi atribuído à remoção das sementes antes da extração, o que pode ter ocorrido também no presente estudo, embora não tenha sido intencional. Ainda, segundo os autores, as sementes de várias plantas são ricas em fenólicos, o que contribui significativamente para a sua atividade antioxidante.

Deepa et al. (2007) encontraram faixa de compostos fenólicos em pimentas verdes de $186-1.122 \mathrm{mg} 100 \mathrm{~g}^{-1}$ (base seca) e em pimentas maduras de 323-852 mg $100 \mathrm{~g}^{-1}$ (base seca), para genótipos de Capsicum provenientes da Índia. Os autores igualmente relataram tendência de aumento de compostos fenólicos ao longo do amadurecimento, para a maioria dos genótipos estudados, à exceção de quatro amostras que apresentaram tendência de queda, com o amadurecimento. Já Conforti et al. (2007), avaliando fenólicos em C. annuum var. acuminatum, observaram teores similares nos dois primeiros estádios de maturação, porém, verificou-se queda significativa, quando as pimentas se mostraram totalmente vermelhas (amadurecidas). No presente estudo, de maneira geral, as pimentas estudadas apresentaram teores significativos de compostos fenólicos, sendo esses teores superiores nas pimentas maduras.

Entre os métodos utilizados para determinar a capacidade de um antioxidante para capturar radicais livres, os radicais $\mathrm{ABTS}^{+}$e $\mathrm{DPPH} \bullet$ são os mais aplicados, por apresentarem elevada sensibilidade, serem práticos, rápidos e muito estáveis (Arnao 2000, Kuskoski et al. 2005).

Observou-se que os nove genótipos de frutos de pimenteira (Tabela 2) mostraram-se eficientes em sequestrar o radical $\mathrm{ABTS}^{+}$, porém, essa ação é diferenciada entre os diferentes genótipos estudados e variou em 55,02-92,03 $\mu \mathrm{M}$ de trolox $\mathrm{g}^{-1}$, para os frutos imaturos, e 46,79-113,06 $\mu \mathrm{M}$ de trolox $\mathrm{g}^{-1}$, para os maduros (Tabela 3). A maior atividade antioxidante foi verificada para o genótipo IAN-186304, no estádio imaturo, e para o genótipo IAN-186311, no estádio maduro. Com relação à comparação entre os estádios de maturação de um mesmo genótipo, diferenças foram observadas entre todos os genótipos, à exceção de IAN-186310 e IAN-186313, com destaque para as pimentas IAN-186304 $(92,03 \mu \mathrm{M}$ de Trolox $\left.\mathrm{g}^{-1}\right)$, no estádio verde, e IAN-186311 $\left(113,08 \mu \mathrm{M}\right.$ de Trolox $\left.\mathrm{g}^{-1}\right)$, para os frutos maduros.

Alvarez-Parrilla et al. (2011), estudando a capacidade antioxidante de pimentas $C$. annuum frescas e processadas, relataram que as pimentas apresentaram alta atividade antioxidante, com valores de 27,76-55,41 $\mu \mathrm{M}$ de Trolox $\mathrm{g}^{-1}$ e destaque para as pimentas frescas, as quais apresentaram valores superiores. Esses resultados estão próximos aos observados para as pimentas IAN-186309, IAN-186312 e IAN-186310 imaturas e para as pimentas IAN186313, IAN-186309, IAN-1863010, IAN-186335 e IAN-186304 maduras. Já para as demais pimentas do presente trabalho, os valores da capacidade antioxidante foram superiores aos encontrados por Alvarez-Parrilla et al. (2011).

Em estudo realizado por Menichini et al. (2009), com pimentas $C$. chinense cv. Habanero, os autores observaram maior atividade antioxidante para os frutos no estádio de maturação verde, ao contrário dos frutos completamente maduros. Já Deepa et al. (2007), avaliando a atividade antioxidante de dez genótipos de pimenta $C$. annuum, verificaram aumento marcante, com o avanço da maturação. Os autores concluíram que ocorrem variações significativas na atividade antioxidante entre diferentes genótipos e estádios de maturação das pimentas. O mesmo foi observado no presente estudo.

A correlação de Pearson, entre cada composto bioativo e sua atividade antioxidante, demonstrou correlação significativa somente entre os compostos fenólicos e a atividade antioxidante pelo método ABTS. Para as análises entre ácido ascórbico e atividade antioxidante e carotenoides totais e atividade antioxidante, as correlações não foram significativas. Para os compostos fenólicos e a atividade antioxidante, a correlação foi alta para os nove genótipos de pimenta estudados, nos dois estádios de maturação, com coeficientes de correlação de 0,86 e 0,95 $(\mathrm{p} \leq 0,05)$, respectivamente para os frutos imaturos (Figura 1a) e maduros (Figura 1b).

Assim como neste estudo, vários outros trabalhos relataram correlação positiva entre a capacidade antioxidante e o teor de compostos fenólicos em frutas e hortaliças (Velioglu et al. 1998, Shan et al. 2005, Dastmalchi et al. 2011). Kapeel et al. (2008), ao avaliarem o conteúdo de compostos fenólicos e propriedades antioxidantes de pimentas Capsicum baccatum L. var. pêndulo, em diferentes estádios de 

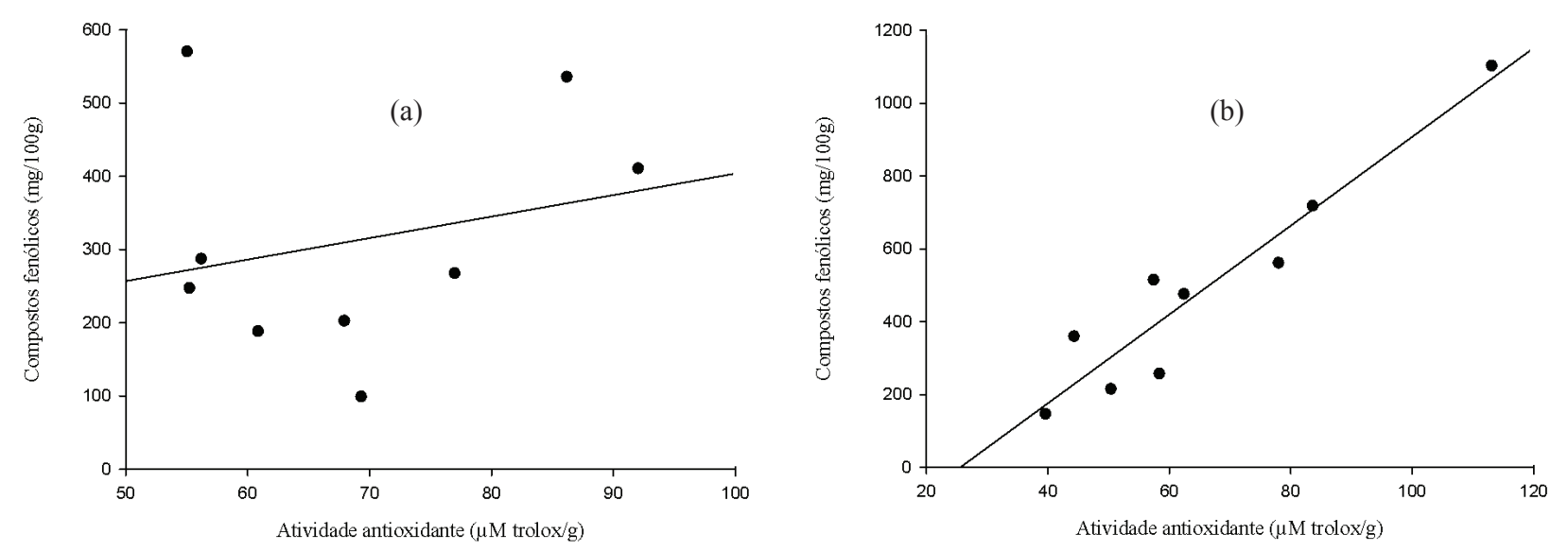

Figura 1. Correlação entre atividade antioxidante e teor de compostos fenólicos totais, em frutos imaturos (a) e maduros (b) de genótipos de pimentas Capsicum spp. (Belém, PA, 2012).

maturação, também encontraram correlação positiva $(\mathrm{r}=0,863)$ para todos os extratos analisados.

A correlação existente entre os compostos fenólicos e a atividade antioxidante sugere que os compostos fenólicos seriam os principais compostos responsáveis pela capacidade antioxidante em pimentas, como relatado por Menichini et al. (2009), que verificaram o mesmo comportamento. No entanto, é importante considerar que, muitas vezes, não só um composto isolado, mas o sinergismo desses compostos naturais presentes nas pimentas, é que proporciona tal potencial antioxidante.

\section{CONCLUSÕES}

1. O grau de maturação influenciou no teor de compostos bioativos dos genótipos de pimenteiras estudados, uma vez que frutos imaturos continham maiores teores de ácido ascórbico e compostos fenólicos, e frutos maduros apresentaram maiores teores de carotenoides totais que os frutos imaturos.

2. Houve forte correlação entre a capacidade antioxidante e o conteúdo de compostos fenólicos, nos genótipos de pimenteira estudados.

3. Destacaram-se como promissores, com potencial para serem utilizados em programas de melhoramento genético, os genótipos IAN-186301 e IAN186324, com relação a altos teores de carotenoides totais; IAN-186301, IAN-186311, IAN-186312 e IAN-186313, com relação a frutos com altos teores de ácido ascórbico; IAN-186304 e IAN-186311, para altos teores de compostos fenólicos; e IAN186311, para atividade antioxidante.

\section{REFERÊNCIAS}

ALVAREZ-PARRILLA, E. et al. Antioxidant activity of fresh and processed Jalapeño and Serrano peppers. Journal of Agricultural and Food Chemistry, Washington, DC, v. 59, n. 1, p. 163-173, 2011.

ASSOCIATION OF OFFICIAL ANALYTICAL CHEMISTS (AOAC). Official methods of analysis of the Association of Official Analytical Chemists. 14. ed. Arlington: AOAC, 1984.

ASSOCIATION OF OFFICIAL ANALYTICAL CHEMISTS (AOAC). Official methods of analysis of the Association of Official Analytical Chemists. 16. ed. Gaithersburg: AOAC, 1997.

ARNAO, M. B. Some methodological problems in the determination of antioxidant activity using chromogen radicals: a practical case. Trends in Food Science and Technology, Cambridge, v. 11, n. 11, p. 419-421, 2000.

BURDA, S.; OLESZEK, W. Antioxidant and antiradical activities of flavonoids. Journal of Agricultural and Food Chemistry, Washington, DC, v. 49, n. 6, p. 2774-2779, 2001.

CARR, A. C.; FREI, B. Toward a new recommended dietary allowance for vitamin $\mathrm{C}$ based on antioxidant and health effects in humans. American Journal of Clinical Nutrition, New York, v. 69, n. 6, p. 1086-107, 1999.

CARVALHO, S. I. C.; BIANCHETTI, L. B. Sistema de produção de pimentas (Capsicum spp.): botânica. Brasília, DF: Embrapa Hortaliças, 2004. (Sistemas de produção, 4).

CHITARRA, A. B. Qualidade, colheita e manuseio póscolheita de frutas do pessegueiro e da ameixeira. Informe Agropecuário, Belo Horizonte, v. 18, n. 189, p. 68-74, 1997. 
CHITARRA, M. I. F.; CHITARRA, A. B. Pós-colheita de frutos e hortaliças: fisiologia e manuseio. 2. ed. Lavras: UFLa, 2005.

CHUAH, A. M. et al. Effect of cooking on the antioxidant properties of coloured peppers. Food Chemistry, Barking, v. 111, n. 1, p. 20-28, 2008.

COLLERA-ZÚÑIGA, O.; JIMÉNEZ, F. G.; GORDILLO, R. M. Comparative study of carotenoid composition in three Mexican varieties of Capsicum annuum L. Food Chemistry, Barking, v. 90, n. 1, p. 109-114, 2005.

CONFORTI, F.; STATTI, G. A.; MENICHINI, F. Chemical and biological variability of hot pepper fruits (Capsicum annuum var. acuminatum $\mathrm{L}$.) in relation to maturity stage. Food Chemistry, Barking, v. 102, n. 4, p. 1096-1104, 2007.

CRUZ, C. D. Programa GENES: biometria. Viçosa: UFV, 2006.

DAVIS, C. B. et al. Determination of capsaicinoids in Habanero peppers by chemometric analysis of UV spectral data. Journal of Agricultural and Food Chemistry, Washington, DC, v. 55, n. 15, p. 5925-5933, 2007.

DASTMALCHI, K. et al. Edible neotropical blueberries: antioxidant and compositional fingerprint analysis. Journal of Agricultural and Food Chemistry, Washington, DC, v. 59, n. 7, p. 3020-3026, 2011.

DEEPA, N. et al. Antioxidant constituents in some sweet pepper (Capsicum annuum L.) genotypes during maturity. LWT - Food Science Technology, London, v. 40, n. 1, p. 121-129, 2007.

FINCO, F. D. B. A. et al. Antioxidant activity and characterization of phenolic compounds from bacaba (Oenocarpus bacaba Mart.) fruit by HPLC-DAD-MS. Journal of Agricultural and Food Chemistry, Washington, DC, v. 60, n. 31, p. 7665-7673, 2012.

GEORGÉ, S. et al. Rapid determination of polyphenols and vitamin $\mathrm{C}$ in plant-derived products. Journal of Agricultural and Food Chemistry, Washington, DC, v. 53, n. 5, p. 1370-1373, 2005.

GODOY, H. T.; RODRIGUEZ-AMAYA, D. B. Occurrence of cis-isomers of provitamin A in Brazilian fruits. Journal of Agricultural and Food Chemistry, Washington, DC, v. 42, n. 6, p. 1306-1313, 1994.

HOWARD, L. R. et al. Changes in phytochemical and antioxidant activity of selected pepper cultivars (Capsicum species) as influenced by maturity. Journal of Agricultural and Food Chemistry, Washington, DC, v. 48, n. 5, p. 17131720, 2000.

KAPPEL, V. D. et al. Phenolic content and antioxidant and antimicrobial properties of fruits of Capsicum baccatum
L. var. pendulum at different maturity stages. Journal of Medicinal Food, New Rochelle, v. 11, n. 2, p. 267-274, 2008.

KAYS, S. J. Postharvest physiology of perishable plant products. New York: Van Nostrand Reinhold, 1991.

KUSKOSKI, E. M. et al. Aplicación de diversos métodos químicos para determinar actividad antioxidante en pulpa de frutos. Ciência e Tecnologia de Alimentos, Campinas, v. 25, n. 4, p. 726-732, 2005.

LARRAURI, J. A.; RUPÉREZ, P.; SAURA-CALIXTO, F. Effect of drying temperature on the stability of polyphenols and antioxidant activity of red grape pomace peels. Journal of Agricultural and Food Chemistry, Washington, DC, v. 45 , n. 4, p. 1390-1393, 1997.

LUTZ, D. L.; FREITAS, S. C. Valor nutricional. In: RIBEIRO, C. S. C. et al. Pimentas Capsicum. Brasília, DF: Embrapa Hortaliças, 2008. p. 31-37.

MARÍN, A. et al. Characterization and quantification of antioxidant constituents of sweet pepper (Capsicum annuum L.). Journal of Agricultural and Food Chemistry, Washington, DC, v. 52, n. 12, p. 3861-3869, 2004.

MÁRKUS, F. et al. Change in the carotenoid and antioxidant content of spice red pepper (paprika) as a function of ripening and some technological factors. Journal of Agricultural and Food Chemistry, Washington, DC, v. 47, n. 1, p. 100-107, 1999.

MATERSKA, M.; PERUCKA, I. Antioxidant activity of the main phenolic compounds isolated from hot pepper fruit (Capsicum annuum L.). Journal of Agricultural and Food Chemistry, Washington, DC, v. 53, n. 5, p. 17501756, 2005.

MENICHINI, F. et al. The influence of fruit ripening on the phytochemical content and biological activity of Capsicum chinense Jacq cv. Habanero. Food Chemistry, Barking, v. 114, n. 2, p. 553-560, 2009.

OGISO, Y. et al. An antioxidant of dried chilli pepper maintained its activity through postharvest ripening for 18 months. Bioscience, Biotechnology, and Biochemistry, Tokyo, v. 72, n. 12, p. 3297-3300, 2008.

RE, R. et al. Antioxidant activity applying an improved ABTS radical cation decolorization assay. Free Radical Biology and Medicine, New York, v. 26, n. 9-10, p. 12311237, 1999.

REIFSCHNEIDER, F. J. B. Capsicum: pimentas e pimentões. Brasília, DF: Embrapa Comunicação para Transferência de Tecnologia, 2000.

RUFINO, M. S. M. et al. Metodologia científica: determinação da atividade antioxidante total em frutas pela captura do radical livre ABTS. Fortaleza: Embrapa Agroindústria Tropical, 2007. (Comunicado técnico, 128). 
SHAN, B. et al. Antioxidant capacity of 26 spice extracts and characterization of their phenolic constituents. Journal of Agricultural and Food Chemistry, Washington, DC, v. 53, n. 20, p. 7749-7759, 2005.

SINGLETON, V. L.; ROSSI JUNIOR, J. A. Colorimetry of total phenolics with phosphomolybdic-phosphotungstic acid reagents. American Journal of Enology Viticulture, Davis, v. 16, n. 1, p. 144-158, 1965.

TOPUZ, A.; OZDEMIR, F. Assessment of carotenoids, capsaicinoids and ascorbic acid composition of some selected pepper cultivars (Capsicum annuum L.) grown in Turkey. Journal of Food Composition and Analysis, San Diego, v. 20, n. 7, p. 596-602, 2007.
VAZQUEZ-OCHOA, R. I.; COLINAS-LEON, M. T. Changes in guavas of three maturity stages in response to temperature and relative humidity. HortScience, Alexandria, v. 25, n. 1, p. 86-87, 1990.

VELIOGLU, Y. S. et al. Antioxidant activity and total phenolics in selected fruits, vegetables, and grain products. Journal of Agricultural and Food Chemistry, Washington, DC, v. 46, n. 10, p. 4113-4117, 1998.

ZHUANG, Y. et al. Bioactive characteristics and antioxidant activities of nine peppers. Journal of Functional Foods, Philadelphia, v. 4, n. 1, p. 331-338, 2012. 